Editorial

2012; 2(3):235-237. doi: 10.7150/thno.4156

\title{
Nano-Carbons as Theranostics
}

\section{Zhuang Liu ${ }^{1 凶}$, Xing-Jie Liang ${ }^{\otimes}$}

1. Institute of Functional Nano \& Soft Materials (FUNSOM), Soochow University, Suzhou, Jiangsu, 215123, China

2. Key Laboratory for Biomedical Effects of Nanomaterials \& Nanosafety, National Center for Nanoscience and Technology, Chinese Academy of Sciences, Beijing, 100190, China

$\triangle$ Corresponding author: zliu@suda.edu.cn, liangxj@nanoctr.cn

(C) Ivyspring International Publisher. This is an open-access article distributed under the terms of the Creative Commons License (http://creativecommons.org/ licenses/by-nc-nd/3.0/). Reproduction is permitted for personal, noncommercial use, provided that the article is in whole, unmodified, and properly cited.

Received: 2012.01.27; Accepted: 2012.01.27; Published: 2012.03.01

\begin{abstract}
Nano-carbons, including fullerenes, carbon nanoparticles, carbon nanotubes, graphene, and nano-diamonds, are an important class of nanostructures attracting tremendous interests in the past two decades. In this special issue, seven review articles and research reports are collected, to summarize and present the latest progress in the exploration of various nano-carbons for theranostic applications.
\end{abstract}

Key words: nano-carbons, biological sensing, imaging, drug delivery, cancer therapies, toxicology

The rapid development in nanoscience and nanotechnology has brought great opportunities to biomedical research in various directions. Among numerous types of nanomaterials developed in the past many years, carbon nanomaterials, or nano-carbons, have attracted particular interests in a large variety of fields including biomedicine [1-5], owing to their highly-enriched distinctive physical and chemical properties.

Based on their bonding structures, nano-carbons may be classified into $\mathrm{sp}^{2}$-carbon nanomaterials and $\mathrm{sp}^{3}$-carbon nanomaterials (Fig. 1). Typical $\mathrm{sp}^{2}$-carbon nanomaterials include zero-dimensional (OD) fuller- ene, one-dimensional (1D) carbon nanotubes (CNTs), and two-dimensional (2D) graphene, all with well-defined structures. Remarkably, the discoveries of fullerene and graphene have been awarded with Nobel prizes in 1996 (Chemistry) and 2010 (Physics), respectively. Carbon nanoparticles or carbon dots (CDots), are nano-clusters of amorphous carbon (or composed by small crystalline structures) with sizes smaller than $10 \mathrm{~nm}$, and may also be deemed as a type of $\mathrm{OD} \mathrm{sp}^{2}$-carbon nanomaterials. $\mathrm{Sp}^{3}$-carbon nanomaterials, on the other hand, usually are nano-diamonds (NDs) with crystal sizes in the nano-range.

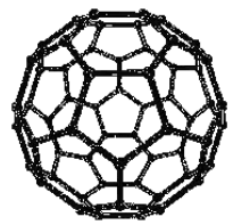

Fullerene

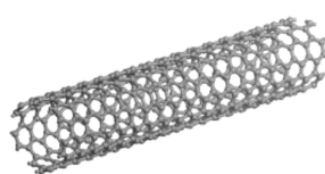

Carbon nanotubes

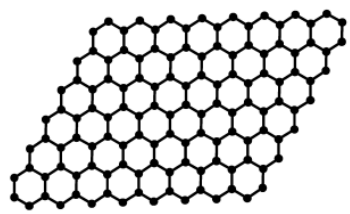

Graphene

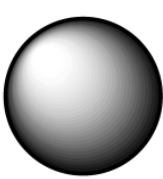

Carbon dots

(Cdots)

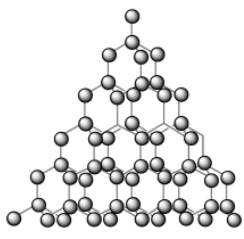

Nano-diamonds

(NDs)

Figure I. Different types of nano-carbons explored for theranostic applications. 
There are many motivations and reasons of using nano-carbons for biomedical applications (e.g. theranostics). Many nano-carbons, including CNTs, graphene derivatives, Cdots, and NDs, show interesting inherent optical properties such as fluorescence, making them useful contrast agents in optical imaging and sensing [6-11]. The excellent electrical properties of CNTs and graphene, on the other hand, allow them to be extensively used in a wide range of biosensing platforms [12-14]. Sp2-carbon nanomaterials especially single-walled carbon nanotubes (SWNTs) and graphene with all carbon atoms exposed on their surfaces, exhibit ultra-high surface area available for efficient drug loading and bioconjugation $[1,10,15,16]$. For fullerene and CNTs with hollow structures, the inside space may be utilized to load other functional species for theranostic purposes[17-20]. CNTs and graphene derivatives with strong optical absorbance in the near-infrared region are also useful for photothermal ablation of cancer[21-25]. Moreover, compared with many other inorganic nanomaterials such as quantum dots (QDs) which usually contain heavy metals, nano-carbons composed simply by carbon are relatively safe at least in term of elementary composition.

In this special issue, we invite colleagues worldwide who have been exploring biomedical applications of nano-carbons for years, to discuss and report cutting-edge research in the use of nano-carbons as theranostics:

In the first article, Chen et al. [26] offer a comprehensive review to summarize the applications of functionalized fullerenes in tumor theranostics. Functionalized fullerenes have not only shown potential in several different cancer therapeutic approaches such as photodynamic therapy, photothermal treatment, radiotherapy and chemotherapy, but also been used as novel contrast agents in magnetic resonance (MR) imaging. The biodistribution, metabolism and toxicity of those functionalized fullerenes are also reviewed in this article.

In the following section, three articles are collected to highlight the promise of using CNTs for potential disease theranostics. Mandal et al. [27] report a label-free real-time electrical detection of whole viruses using CNT think film field effect devices, and achieve an ultra-high sensitivity which is 5 orders of magnitude higher than traditional electrical impedance sensors without CNTs. In the next article, $\mathrm{Xu}$ and co-workers [28] present an interesting study to investigate the influence of oxidized multi-walled carbon nanotubes (o-MWNTs) on marcophages. It is uncovered that subcutaneous injection of o-MWNTs would lead to macrophage reduction and decreased vessel density in the tumor by competing with the tumor mass for recruitment of macrophages from circulating monocytes, resulting in the inhibition of tumor progression and metastasis. This finding presents a novel approach of using CNTs in cancer immunotherapy. Besides these two research articles, a review article is contributed by Yang et al. [29] to discuss the pharmacokinetics, metabolism and toxicity of CNTs in details. Surveying others reports and the authors' own findings, it is summarized that the surface chemistry of CNTs is crucial in regulating the in vivo behaviors of CNTs. Compared with non-functionalized CNTs, well functionalized CNTs (e.g. by PEGylation) by both covalent and non-covalent methods are much less toxic in vitro and in vivo, and may be preferred for applications in biomedicine. However, the authors also point out that future studies are still needed to systematically evaluate long-term toxicity of CNT-exposure. Establishing fast and reliable analytical tools to study in vivo behaviors of CNTs as well as other newly developed nanomaterials is also of great importance.

Graphene has been a shining star in materials science since 2004. Its applications in biomedicine have also aroused widespread interest. In the article by Zhang and co-workers [30], the authors summarize the latest progress of applying graphene and graphene derivatives in various directions of biomedicine including drug and gene delivery, cancer therapies, biomedical imaging, biosensing, as well as tissue engineering scaffolds. The opportunities and challenges in this rapidly developing field are also discussed.

Cdots are surface-functionalized small carbon nanoparticles, which with certain modification, would emit bright fluorescence useful in biomedical imaging. In the article by Sun and co-workers [31], highly fluorescent Cdots without and with ZnS doping are prepared and evaluated for optical imaging in mice. Significantly, competitive performance of Cdots in vivo to that of well-established CdSe/ZnS QDs is observed, suggesting that Cdots may be a new class of non-heavy-metal containing optical probes promising in bioimaging.

In additions to $\mathrm{sp}^{2}$-carbon nanomaterials, NDs with $\mathrm{sp}^{3}$-bonded structure have also been extensively explored in nanomedicine. The last article of this issue is contributed by Zhu et al. [32] to review the in vitro and in vivo toxicity of NDs as well as their applications in drug delivery systems. With rather different properties and behaviors from $\mathrm{sp}^{2}$ carbon nanomaterials such as CNTs and graphene, NDs and ND-clusters appear to be relatively safe in biological systems and are also potential candidates as drug 
carriers for fluorescent imaging guided delivery.

In summary, the development of novel theranostic approaches based on nano-carbons has been and would remain to be an area attracting great attention in the field of nanomedicine. This special issue containing 4 review articles and 3 original research reports covers all widely explored nano-carbons in biomedicine. At this point, we would like to thank all the authors for their excellent contribution in this project, and hope that our issue would be informative and beneficial for researchers working in or interested in this area!

\section{Conflict of Interest}

The authors have declared that no conflict of interest exists.

\section{References}

1. Liu Z, Robinson JT, Tabakman SM, et al. Carbon materials for drug delivery \& cancer therapy. Mater Today. 2011; 14: 316-323.

2. Feng LZ, Liu ZA. Graphene in biomedicine: opportunities and challenges. Nanomedicine. 2011; 6: 317-324.

3. Liu Z, Tabakman SM, Chen Z, et al. Preparation of carbon nanotube bioconjugates for biomedical applications. Nat Protoc. 2009; 4: 1372-1382.

4. Liu Z, Tabakman S, Welsher $\mathrm{K}$, et al. Carbon nanotubes in biology and medicine: in vitro and in vivo detection, imaging and drug delivery. Nano Res. 2009; 2: 85-120.

5. Kostarelos K, Bianco A, Prato M. Promises, facts and challenges for carbon nanotubes in imaging and therapeutics. Nat Nanotechnol. 2009; 4: 627-633.

6. Welsher K, Liu Z, Sherlock SP, et al. A route to brightly fluorescent carbon nanotubes for near-infrared imaging in mice. Nat. Nanotech. 2009; 4: 773-780.

7. Liu Z, Yang K, Lee ST. Single-walled carbon nanotubes in biomedical imaging. J Mater Chem. 2011; 21: 586-598.

8. Sun YP, Zhou B, Lin Y, et al. Quantum-sized carbon dots for bright and colorful photoluminescence. J Am Chem Soc. 2006; 128: 7756-7757.

9. Yu SJ, Kang MW, Chang HC, et al. Bright fluorescent nanodiamonds: no photobleaching and low cytotoxicity. J Am Chem Soc. 2005; 127: 17604-17605.

10. Sun X, Liu Z, Welsher K, et al. Nano-graphene oxide for cellular imaging and drug delivery. Nano Res. 2008; 1: 203-212.

11. Loh KP, Bao Q, Eda G, et al. Graphene oxide as a chemically tunable platform for optical applications. Nat Chem. 2010; 2: 1015-1024.

12. Chen RJ, Bangsaruntip S, Drouvalakis KA, et al. Noncovalent functionalization of carbon nanotubes for highly specific electronic biosensors. Proc Natl Acad Sci U S A. 2003; 100: 4984-4989.

13. Yang W, Ratinac KR, Ringer SP, et al. Carbon nanomaterials in biosensors: should you use nanotubes or graphene? Angew Chem Int Ed Engl. 2010; 49: 2114-2138.

14. Mohanty N, Berry V. Graphene-based single-bacterium resolution biodevice and DNA transistor: interfacing graphene derivatives with nanoscale and microscale biocomponents. Nano Lett. 2008; 8: 4469-4476.

15. Liu Z, Sun X, Nakayama N, et al. Supramolecular chemistry on water-soluble carbon nanotubes for drug loading and delivery. ACS Nano. 2007; 1: 50-56.
16. Liu Z, Robinson JT, Sun XM, et al. PEGylated nanographene oxide for delivery of water-insoluble cancer drugs. J Am Chem Soc. 2008; 130: 10876-10877.

17. Chen C, Xing G, Wang J, et al. Multihydroxylated [Gd@C82(OH)22]n nanoparticles: antineoplastic activity of high efficiency and low toxicity. Nano Lett. 2005; 5: 2050-2057.

18. Liang XJ, Meng H, Wang Y, et al. Metallofullerene nanoparticles circumvent tumor resistance to cisplatin by reactivating endocytosis. Proc Natl Acad Sci U S A. 2010; 107: 7449-7454.

19. Meng H, Xing G, Sun B, et al. Potent angiogenesis inhibition by the particulate form of fullerene derivatives. ACS Nano. 2010; 4 : 2773-2783.

20. Hong SY, Tobias G, Al-Jamal KT, et al. Filled and glycosylated carbon nanotubes for in vivo radioemitter localization and imaging. Nat Mater. 2010; 9: 485-490.

21. Yang K, Wan J, Zhang S, et al. The influence of surface chemistry and size of nanoscale graphene oxide on photothermal therapy of cancer using ultra-low laser power. Biomaterials. 2012; 33: 2206-2214.

22. Yang K, Zhang S, Zhang G, et al. Graphene in mice: ultra-high in vivo tumor uptake and photothermal therapy. Nano Lett. 2010; 10: 3318-3323.

23. Liu $X$, Tao $H$, Yang $K$, et al. Optimization of surface chemistry on single-walled carbon nanotubes for in vivo photothermal ablation of tumors. Biomaterials. 2011; 32: 144-151.

24. Robinson JT, Welsher K, Tabakman SM, et al. High performance in vivo near-IR (>1 mu $\mathrm{m}$ ) imaging and photothermal cancer therapy with carbon nanotubes. Nano Res. 2010; 3: 779-793.

25. Moon HK, Lee SH, Choi HC. In vivo near-infrared mediated tumor destruction by photothermal effect of carbon nanotubes. Acs Nano. 2009; 3: 3707-3713.

26. Chen Z, Ma L, Liu Y, et al. Applications of functionalized fullerenes in tumor theranostics. Theranostics. 2012; 2: 238-250

27. Mandal HS, Su Z, Ward A, et al. Carbon nanotube thin film biosensors for sensitive and reproducible whole virus detection. Theranostics. 2012; 2: 251-7

28. Yang M, Meng J, Cheng X, et al. Multiwalled carbon nanotubes interact with macrophages and influence tumor progression and metastasis. Theranostics. 2012; 2: 258-70

29. Yang S-T, Luo J, Zhou Q, et al. Pharmacokinetics, metabolism and toxicity of carbon nanotubes for biomedical purposes. Theranostics. 2012; 2: 271-82

30. Shen H, Zhang L, Liu M, et al. Biomedical applications of graphene. Theranostics. 2012; 2: 283-94

31. Cao L, Yang S-T, Wang $X$, et al. Competitive performance of carbon "quantum" dots in optical bioimaging. Theranostics. 2012; 2: 295-301

32. Zhu Y, Li J, Li W, et al. The biocompatibility of nanodiamonds and their application in drug delivery systems. Theranostics. 2012; 2: 302-12 